\title{
Phonon dispersion and electron-phonon interaction in peanut-shaped fullerene polymers
}

\author{
Shota Ono* and Hiroyuki Shima \\ Division of Applied Physics, Faculty of Engineering, \\ Hokkaido University, Sapporo, Hokkaido 060-8628, Japan
}

(Dated: November 9, 2018)

\begin{abstract}
We reveal that the periodic radius modulation peculiar to one-dimensional (1D) peanut-shaped fullerene $\left(\mathrm{C}_{60}\right)$ polymers exerts a strong influence on their low-frequency phonon states and their interactions with mobile electrons. The continuum approximation is employed to show the zonefolding of phonon dispersion curves, which leads to fast relaxation of a radial breathing mode in the $1 \mathrm{D} \mathrm{C}_{60}$ polymers. We also formulate the electron-phonon interaction along the deformation potential theory, demonstrating that only a few set of electron and phonon modes yields a significant magnitude of the interaction relevant to the low-temperature physics of the system. The latter finding gives an important implication for the possible Peierls instability of the $\mathrm{C}_{60}$ polymers suggested in the earlier experiment.
\end{abstract}

PACS numbers: 72.80.Rj, 63.22.-m, 63.20.kd

\section{INTRODUCTION}

Successful syntheses of fullerene $\left(\mathrm{C}_{60}\right)$ molecules and related nanomaterials 1, 2] have inspired tremendous interest in the community of nanotechnology and nanoscience. Experimental studies have shown that electron-beam irradiation to a $\mathrm{C}_{60}$ film induces polymerizations and produces one-dimensional (1D) $\mathrm{C}_{60}$ polymers [3, 4]. The polymers have peanut-like geometry, i.e., the tube structure with periodically modulated radius along the axis [5-9]. Such the peanut structure was confirmed by comparison between the infrared spectral measurement [3] and the first-principle density-functional calculations of the vibrational spectra [7, 9]. Recently, it was predicted that the periodic radius modulation strongly affects transport properties of the carrier in the 1D $\mathrm{C}_{60}$ polymers [10]. Similar periodic corrugation of a two-dimensional conducting film was also shown to increase the electrical resistivity [11]. These findings are attributed to an effective electrostatic field caused by the periodic corrugation, which gives rise to a Fermi velocity shift and electron-electron scattering enhancement.

The $1 \mathrm{D} \mathrm{C}_{60}$ polymers exhibit semiconducting or metallic nature depending on the irradiation time interval [4]. This suggests that well-controlled irradiation time may lead the fabrication of quantum wires with desired conductivity. Of particular interest is the metallic $\mathrm{C}_{60}$ polymers; in the metallic cases, nonequiliblium photo-excited carriers exhibit anomalously slow relaxation below $60 \mathrm{~K}$, indicating pseudogap formation in the electron energy band near the Fermi level [12]. Phenomenologically, this pseudogap is likely to result from the Peierls instability associated with a periodic distortion of the underlying carbon structure. However, its theoretical understanding has not been attained; in fact, it has remained unclear how the periodic radius modulation in the $\mathrm{C}_{60}$ polymers affects the phononic excitations and electron-phonon couplings in the systems.

We point out that the periodic geometry of the $1 \mathrm{D}$
$\mathrm{C}_{60}$ polymers will induce a pronounced shift in the lowfrequency phonon spectrum where the phonon wavelength is comparable to the radius modulation period, i.e., larger enough than the carbon bond length. In the low-frequency range, continuum elastic approximation is a powerful means to analyze the phononic excitations. Furthermore, the approximation allows to use the deformation potential theory 13] that describes the electronphonon interaction in a conducting medium. Combining the two methods, therefore, we can formulate the radius modulation effect on the phonon spectrum and the electron-phonon interaction in the $1 \mathrm{D}_{60}$ polymers; the results obtained give a fundamental clue to explore novel properties of collective excitations in the system.

In this paper, we study the low-frequency phonons and their coupling with electrons in the $1 \mathrm{D} \mathrm{C}_{60}$ polymers by using a continuum model. A generalized eigenvalue equation is formulated to obtain phonon dispersion relations that show zone-folding due to the periodic geometry. It is demonstrated that only a particular set of phonon modes, among many choices, yields marked enhancement in the electron-phonon coupling strength; these specific phonon modes are thought to play a prominent role in the possible Peierls instability in the $1 \mathrm{D} \mathrm{C}_{60}$ polymers. We also show that the zone-folding in the phonon spectrum encourages the relaxation of radial breathing modes in the $1 \mathrm{D} \mathrm{C}_{60}$ polymers, which is a consequence of the periodic radius modulation.

In Sec. IIA we derive an eigenvalue equation that is useful for calculating eigenmodes of general isotropic elastic medium with cylindrical symmetry. In Sec. IIB, we introduce a continuum elastic model of the $1 \mathrm{D} \mathrm{C}_{60}$ polymers and suitable basis functions for an efficient eigenmode analysis: Appropriate values of the elastic constants and geometric parameters of the model are presented in Sec. IIC Section III shows the zone-folding of phonon dispersion curves caused by the periodic geometry. Section. [V] is devoted to formulate the electronphonon interaction Hamiltonian on the basis of the de- 


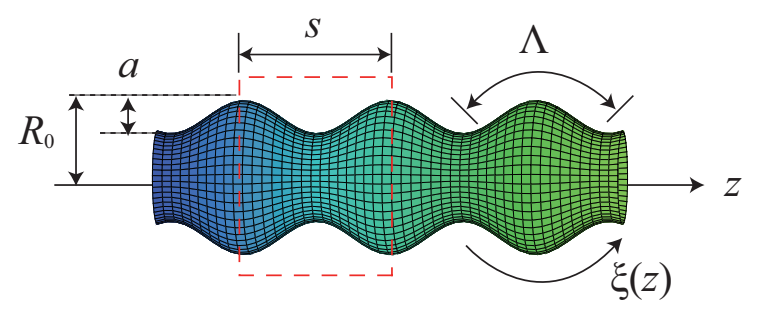

FIG. 1. (Color online) Continuum model of a 1D peanutshaped $\mathrm{C}_{60}$ polymer. The dashed square (red in color) indicates the unit cell.

formation potential theory. Numerical results of the coupling strength are shown in Sec. V] and associated physical implications are discussed in Sec. VI The paper is closed by conclusion given in Sec. VII

\section{CONTINUUM ELASTIC MODEL}

In this section, we formulate a continuum elastic model of the $1 \mathrm{D} \mathrm{C}_{60}$ polymers. Thus far, several continuum models have been developed to study low-frequency phonons in nanostructures such as GaAs/AlAs based nanowires [14, 15], pristine $\mathrm{C}_{60}$ molecules [16], and carbon nanotubes [17-20]. Our model discussed below takes into account the effects of the periodic structure on the vibrational properties of the system, and the resultant vibrational eigenmodes will be applied for defining the electron-phonon interaction Hamiltonian in Sec. IV.

\section{A. EIGENVALUE EQUATION}

The outline below is our derivation of a generalized eigenvalue equation [i.e., Eq. (4)] that allows to obtain the vibrational eigenmodes of an elastic medium. For general cylindrical objects, elastic vibration is governed by the Lagrangian:

$$
\mathcal{L}=\int_{V}\left(\frac{\rho \omega^{2}}{2} \sum_{i} u_{i}^{*} u_{i}-\frac{\lambda}{2} \Delta^{*} \Delta-\mu \sum_{i} \sum_{j} e_{i j}^{*} e_{i j}\right) d V .
$$

Here, $\rho$ is the mass density, $\omega$ is the vibrational frequency, $\lambda$ and $\mu$ are Lame coefficients. The complex-valued function $u_{i}$ denotes the $i$ th component of the displacement vector $\boldsymbol{u}(\boldsymbol{r})$ at a point $\boldsymbol{r}$, and $u_{i}^{*}$ is its complex conjugate. Using the cylindrical coordinates, we can write
$\Delta \equiv e_{r r}+e_{\theta \theta}+e_{z z}$ with the strain tensors [21]:

$$
\begin{aligned}
& e_{r r}=\partial_{r} u_{r}, \quad e_{\theta \theta}=\frac{1}{r} \partial_{\theta} u_{\theta}+\frac{u_{r}}{r}, \quad e_{z z}=\partial_{z} u_{z}, \\
& e_{r \theta}=e_{\theta r}=\frac{1}{2}\left(\partial_{r} u_{\theta}-\frac{u_{\theta}}{r}+\frac{1}{r} \partial_{\theta} u_{r}\right), \\
& e_{\theta z}=e_{z \theta}=\frac{1}{2}\left(\frac{1}{r} \partial_{\theta} u_{z}+\partial_{z} u_{\theta}\right) \\
& e_{z r}=e_{r z}=\frac{1}{2}\left(\partial_{z} u_{r}+\partial_{r} u_{z}\right),
\end{aligned}
$$

where $\partial_{i}$ means the partial derivative with respect to the variables $i=r, \theta, z$. The integral in Eq. (1) should be taken over the whole elastic medium. The first term of the integrand in Eq. (1) represents the kinetic energy density, and the other two terms account for the potential energy density with opposite sign.

To the aim, we impose stress-free boundary conditions on the whole surface of the medium and follow the procedure described in Ref. [22]. Expand $u_{i}$ by a set of basis functions $\phi_{\alpha}(\boldsymbol{r})$ :

$$
u_{i}(\boldsymbol{r})=\sum_{\alpha} A_{\alpha i} \phi_{\alpha}(\boldsymbol{r})
$$

where $\alpha$ labels a basis function. Preferred forms of $\phi_{\alpha}(\boldsymbol{r})$ depend on the shape of the medium considered, and those for the present study will be given in Eq. (9). Substituting Eq. (3) into Eq. (11) and employing the variation method with respect to $A_{\alpha i}$, we obtain the generalized eigenvalue equation:

$$
\sum_{\alpha, j} \Gamma_{(\beta i),(\alpha j)} A_{\alpha j}=\omega^{2} \sum_{\alpha, j} E_{(\beta i),(\alpha j)} A_{\alpha j} .
$$

The matrix elements of $E_{(\beta i),(\alpha l)}$ have a simple form written as

$$
E_{(\beta i),(\alpha j)}=\delta_{i j} \frac{1}{V} \int_{V} \phi_{\beta}^{*} \phi_{\alpha} d V .
$$

Expressions of $\Gamma_{(\beta i),(\alpha l)}$ are somewhat complicated, thus summarized in Appendix A.

\section{B. HOLLOW TUBE WITH MODULATED RADIUS}

Next we restrict our attention to elastic thin hollow tubes with thickness $2 h$ and length $L$, which we consider as a continuum model of the $1 \mathrm{D} \mathrm{C}_{60}$ polymers. The tube axis points to the $z$ direction, and the tube radius is spatially modulated as

$$
R(z)=R_{0}-\frac{a}{2}+\frac{a}{2} \cos \left(\frac{2 \pi z}{s}\right),
$$

where $R_{0}$ and $R_{0}-a$ are the maximum and minimum radii, respectively. Since $R(z)$ is periodic with a period $s$, we can define a unit cell by a portion of the tube within 
the region $z \in[0, s]$ as depicted in Fig. 1] For later use, we introduce a new variable $\xi(z)$ defined by

$$
\xi(z)=\int_{0}^{z} w\left(z^{\prime}\right) d z^{\prime}, \quad w(z)=\sqrt{1+\left(\partial_{z} R\right)^{2}} .
$$

Here, $\xi(z)$ measures the length of a geodesic curve [23] between two points along the curved membrane represented in Fig[1] In terms of $\xi(z)$, a unit cell of the tube is a portion within the region $\xi \in[0, \Lambda]$ where $\Lambda=\xi(s)$.

Once we specify the system's geometry as above, the volume integral in Eq. (5) is written by

$$
\int_{V} d V=\int_{0}^{L} d z \int_{R(z)-h}^{R(z)+h} r d r \int_{0}^{2 \pi} d \theta .
$$

To eliminate the finite length effect, we suppose $u_{i}(r, \theta, z)=u_{i}(r, \theta, z+L)$ with $L=N s$, where $N$ is the number of unit cells and $N=800$ in our calculation. Under the condition, the wavenumber $q$ along the $z$ direction serves as an additional index (complementary to the label $\alpha$ ) that specifies phonon modes; here $q=2 \pi p /(N \Lambda)$ with $-N / 2<p \leq N / 2$ ( $p$ is an integer). The basis function with $q$ and $\alpha$ is defined by

$$
\phi_{q, \alpha}(\boldsymbol{r})=\left(\frac{r}{R_{0}}\right)^{l} e^{i m \theta} e^{i(q-G) \xi},
$$

where $l=0,1,2, \cdots l_{\max }, m=0, \pm 1, \pm 2, \cdots$, and $G=$ $2 \pi n / \Lambda(n=0, \pm 1, \pm 2, \cdots)$. We used in Eq. (9) the expression of $e^{i q \xi}$ rather than $e^{i q z}$, since phonons propagate along the $\xi$ axis, not the $z$ axis. Expression (9) implies that the label $\alpha$ represents the set of three integers: $(l, m, n)$.

It is noteworthy that $\phi_{q, \alpha}$ with $m$ and $\phi_{q, \beta}^{*}$ with $m^{\prime}(\neq$ $m)$ are orthogonal in the sense of the volume integral (8). Therefore, individual phonon dispersion curves in the $q$ $\omega$ plane are obtained by solving the eigenvalue equation (4) with $m$ being fixed.

\section{NUMERICAL PARAMETERS}

In the phonon mode analysis, it is essential to determine appropriate values of the Lame coefficients $\lambda, \mu$ and the mass density $\rho$ introduced in Eq.(11). The Lame coefficients are expressed by 21]

$$
\lambda=\frac{\nu E}{(1+\nu)(1-2 \nu)}, \quad \mu=\frac{E}{2(1+\nu)},
$$

where $E$ and $\nu$ are the Young modulus and the Poisson ratio of the system, respectively. To our knowledge, there has been no attempt of determining $E, \nu$, and $\rho$ of $1 \mathrm{D}$ peanut-shaped $\mathrm{C}_{60}$ polymers. Meanwhile, pristine $\mathrm{C}_{60}$ molecules were suggested to have $E=1.06 \mathrm{TPa}$, $\nu=0.145$, and $\rho=2.27 \mathrm{~g} / \mathrm{cm}^{3}$, whose values are similar to those of graphene sheets [16]. We postulate that the three material constants of the $1 \mathrm{D}_{60}$ polymer show (a)

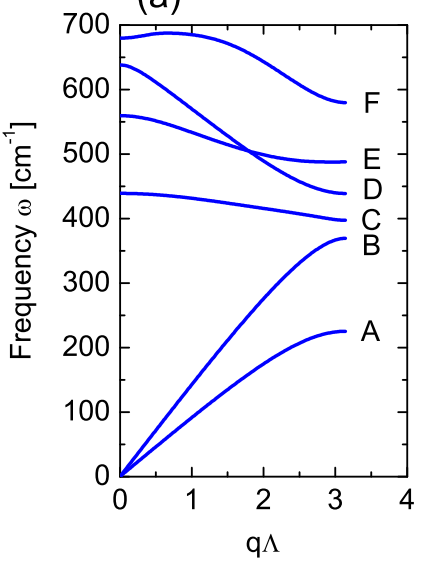

(b)

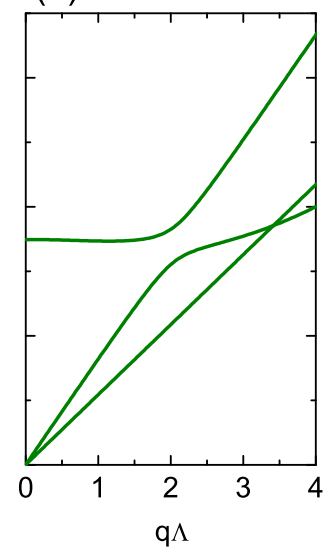

FIG. 2. (Color online) Phonon dispersion relations of hollow cylinders with periodically varying radius (a) and those with constant radius (b). The angular momentum index $m$ is set to be $m=0$ for both plots. Six capitals (A-F) are assigned to the eigenmodes at $q \Lambda=\pi$ for each dispersion curve in (a).

the similar values to those noted above, motivated by the fact that adjacent $\mathrm{C}_{60}$ molecules in the polymer are connected via the $\sigma$ bonds as same as adjacent carbon atoms within a pristine $\mathrm{C}_{60}$ molecule.

Given below is the list of geometric parameters we have used: $R_{0}=3.5 \AA, s=7.0 \AA$, and $a=1.5 \AA$, all of which were referred to the experimental characterization of the $1 \mathrm{D} \mathrm{C}_{60}$ polymers discussed in Ref. [9]. The tube thickness is fixed to be $2 h=3.2 \AA$ as derived from the relation $\rho=M_{t} / v$, where $M_{t}=1.2 \times 10^{-24} \mathrm{~kg}$ is the total mass of 60 carbon atoms and $v=4 \pi\left[\left(R_{0}+h\right)^{3}-\left(R_{0}-h\right)^{3}\right] / 3$ is the volume of a hollow spherical shell with thickness $2 h$ that corresponds to the pristine $\mathrm{C}_{60}$ atomic structure when it is smeared out.

Before addressing the polymer's dispersion, we have checked the validity of our formulation by applying it to a straight hollow cylinder with no radius modulation. The exact solution of the vibrational eigenmode in the straight cylinder is $e^{i(m \theta+q z)}$ multiplied by $r$-dependent Bessel functions (and their derivatives) [19]. In our formulation, the $r$-dependent Bessel terms are approximated by a set of polynomial functions, as seen from Eqs. (3) and (9). Despite this approximation, the computed phonon frequencies were in agreement with the exact solution to more than $99 \%$ accuracy when we set $l_{\max }=6$. The same value of $l_{\max }$ has been used in the calculations of the modulated cylinders (i.e., the $1 \mathrm{D} \mathrm{C}_{60}$ polymers), wherein 35 basis functions were employed to solve the eigenvalue equation (4); in fact, we set $0 \leq l \leq 6$ and $-2 \leq n \leq 2$ for each fixed $m$. It should be noted that for very high frequency range $\left(\omega>700 \mathrm{~cm}^{-1}\right.$ in our study; see Sec. (III), $l_{\max }$ must be larger than our choice, but such the high frequency range is beyond the scope of the present analysis. 


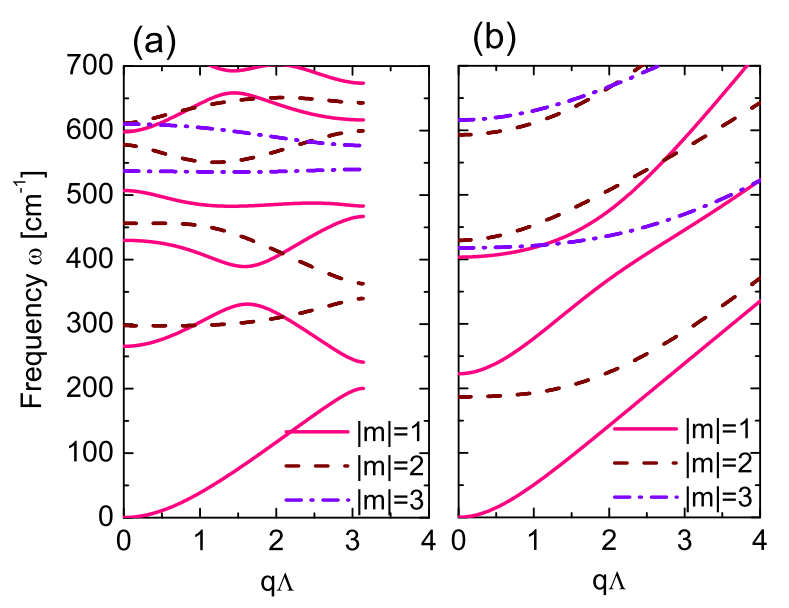

FIG. 3. (Color online) Dispersion relations for $|m|=1,2,3$ of hollow cylinders with: (a) periodically varying radius and (b) constant radius.

\section{DESPERSION RELATIONS AND MODE ANALYSIS}

Figure 2(a) shows the lowest six phonon dispersion curves for $m=0$ in the modulated tube. For comparison, we plot in Fig. 2(b) the lowest three curves for the straight tube with radius $3.5 \AA$; the six curves in Fig. 2(a) reduce to the three ones in Fig. 2(b) if we set $a=0 \AA$ [see Eq. (6)]. In the low frequency range $\left(\omega<200 \mathrm{~cm}^{-1}\right)$, the polymer's dispersion relations are almost identical to those of the straight tube; two almost-linear dispersions are present. Among the two, the bottom curve belongs to a twisting mode $\left(\omega=c_{t} q, c_{t}=\sqrt{\mu / \rho} \simeq\right.$ $14.3 \mathrm{~km} / \mathrm{s})$, and the upper to a longitudinal mode $(\omega=$ $\left.c_{l} q, c_{l}=\sqrt{(\lambda+2 \mu) / \rho} \simeq 22.1 \mathrm{~km} / \mathrm{s}\right)$. Similar two linear branches were found in carbon nanotubes through continuum [18, 19] and atomistic calculations [24, 25], while the magnitude of frequencies deviates from the present results. An important observation in Fig. 2(a) and2(b) is a significant difference in the dispersion profiles at intermediate frequencies $\left(200 \mathrm{~cm}^{-1}<\omega<700 \mathrm{~cm}^{-1}\right)$ between the polymer and the straight tube. The periodic radius modulation causes zone-folding at $q \Lambda=\pi$, resulting in additional branches in this frequency range. This implies that the radius modulation effect can be traced by investigating the phononic properties in the branches.

Figures 3(a) and 3(b) show the phonon dispersion relations of the modulated and straight tubes, respectively, for $|m|=1,2,3$. Similarly to the case of $m=0$, zonefolding at $q \Lambda=\pi$ and marked difference in the dispersion profiles at the intermediate frequencies are observed. In the following, we specify all the vibrational states in the plots by three indexes: $q, m$, and the band index $M=1,2, \cdots$, which labels the $M$ th lowest curve for each $m$.

Figure 4 shows the displacement distribution in the

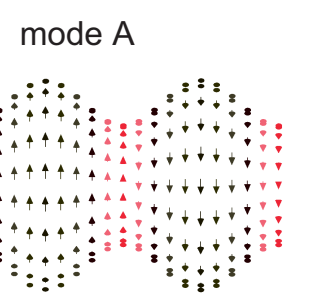

mode $B$

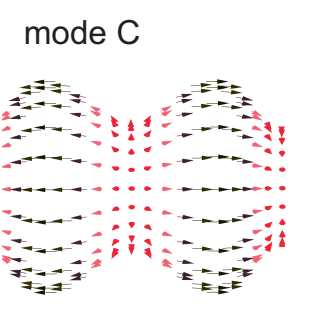

mode D

mode $\mathrm{E}$

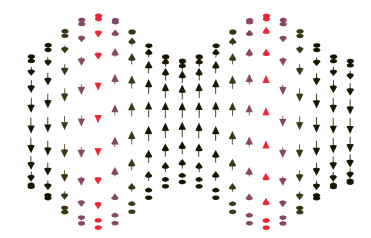

mode $\mathrm{F}$
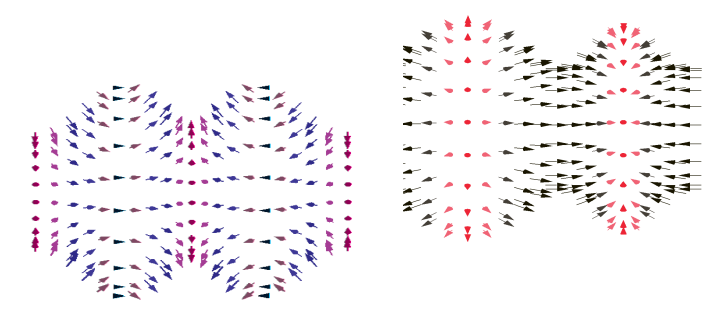

FIG. 4. (Color online) Displacement distribution of the lowest six modes at $q \Lambda=\pi$ within the region $z \in[s / 2,5 s / 2]$. The magnitude of displacement vectors is signified by the lengths and colors of arrows (red arrows are longer than blue ones in color).

vibrational modes at $q \Lambda=\pi$ for each six curves presented in Fig. 2(a). The profiles within the region $z \in[s / 2,5 s / 2]$ are picked up for all the six modes that we labeled by $\mathrm{A}-\mathrm{F}$ in order of increasing the eigenfrequency. The modes $\mathrm{A}$ and $\mathrm{D}$ correspond to twisting modes in which all vectors are parallel to the $\theta$ direction. The other four are hybridized modes of longitudinal and radial breathing modes, where vectors point to directions in-between the $z$ and $r$ axes.

\section{ELECTRON-PHONON INTERACTION}

In view of electron-phonon couplings, the hybridized modes of $\mathrm{C}$ and $\mathrm{F}$ depicted in Fig. 4 give an important consequence. To explain it, we formulate in this section the interaction Hamiltonian between electrons and lowfrequency phonons along the deformation potential theory [13]. The theory states that low-frequency phonons perturb electronic states through the occurrence of a deformation potential represented by $D \nabla \cdot \tilde{\boldsymbol{u}}$. Here, $D$ is the deformation potential constant and $\tilde{\boldsymbol{u}}$ denotes the normalized displacement vector. The interaction Hamil- 
tonian is given by

$$
\mathcal{H}_{\mathrm{e}-\mathrm{ph}}=\int \Psi^{\dagger}(\theta, z)[D \nabla \cdot \tilde{\boldsymbol{u}}]_{r=R(z)} \Psi(\theta, z) d S,
$$

where $\Psi\left(\Psi^{\dagger}\right)$ represents the annihilation (creation) operator for electrons. The value of $D$ lies within the range $10-30 \mathrm{eV}$ for various nanocarbon materials [26, 27], although its exact value for the $1 \mathrm{D} \mathrm{C}_{60}$ polymer has not yet been examined; we thus set $D=20 \mathrm{eV}$ in the present work. The surface integral in Eq. (11) is written as

$$
\int d S=\int_{0}^{2 \pi} d \theta \int_{0}^{N s} d z R(z) w(z) .
$$

We now derive an alternative form of $\mathcal{H}_{\mathrm{e}-\mathrm{ph}}$ [i.e., Eq. (18)] by expanding the field operators $\tilde{\boldsymbol{u}}$ and $\Psi$ introduced in Eq. (11). First, the $i$ th component of the quantized phonon field $\tilde{\boldsymbol{u}}$ is expressed by

$$
\tilde{u}_{i}=\sum_{J}\left(b_{J} u_{J, i}+b_{J}^{\dagger} u_{J, i}^{*}\right)
$$

where $b_{J}\left(b_{J}^{\dagger}\right)$ is the phonon annihilation (creation) operator and $J$ denotes the mode index of phonons, i.e., $\omega_{J}=\omega_{m, q, M}$. The $u_{J, i}$ in Eq. (13) is written as

$$
u_{J, i}=c_{J} \sum_{\alpha} A_{\alpha i}^{(J)} \phi_{\alpha}(\boldsymbol{r})
$$

Following the quantization procedure [28], $c_{J}$ should be determined by carrying out the integral:

$$
\int \boldsymbol{u}_{J^{\prime}}^{*} \cdot \boldsymbol{u}_{J} d V=\frac{\hbar}{2 \rho \omega_{J}} \delta_{J, J^{\prime}}
$$

Consequently, we obtain the normalization constants:

$$
c_{J}=\frac{1}{\sqrt{\boldsymbol{A}^{(J) \dagger} \cdot \boldsymbol{E} \cdot \boldsymbol{A}^{(J)}}} \sqrt{\frac{\hbar}{2 \rho V \omega_{J}}},
$$

where $\boldsymbol{A}^{(J)}$ is the eigenvector corresponding to $\omega_{J}, \boldsymbol{E}$ is the matrix defined in Eq. (4), $V$ is the volume of the system.

Next, the field operator for the electron is written as

$$
\Psi(\theta, z)=\sum_{\gamma, k, H, \sigma} a_{\gamma, k, \sigma}^{(H)} \psi_{\gamma, k}^{(H)}(\theta, z),
$$

where $a_{\gamma, k, \sigma}^{(H)}$ is the annihilation operator of the electron with spin $\sigma, \gamma$ is the angular quantum number, $k$ is the wavenumber, and $H$ is the band index. Explicit forms of the electron's eigenfunctions $\psi_{\gamma, k}^{(H)}(\theta, z)$ are derived in Appendix B The electronic band structure $\epsilon_{\gamma}^{(H)}$ as a function of $k$ is obtained by numerical diagonalizations of the Schrödinger equation (B1). The lowest few electronic dispersion curves $\epsilon_{\gamma}^{(H)}(k)$ for several $\gamma$ and $H$ are drawn in Fig. [5. Zone-folding at $k \Lambda= \pm \pi$ is due to the curvatureinduced electrostatic potential with the same periodicity of the radius modulation [10, 29].

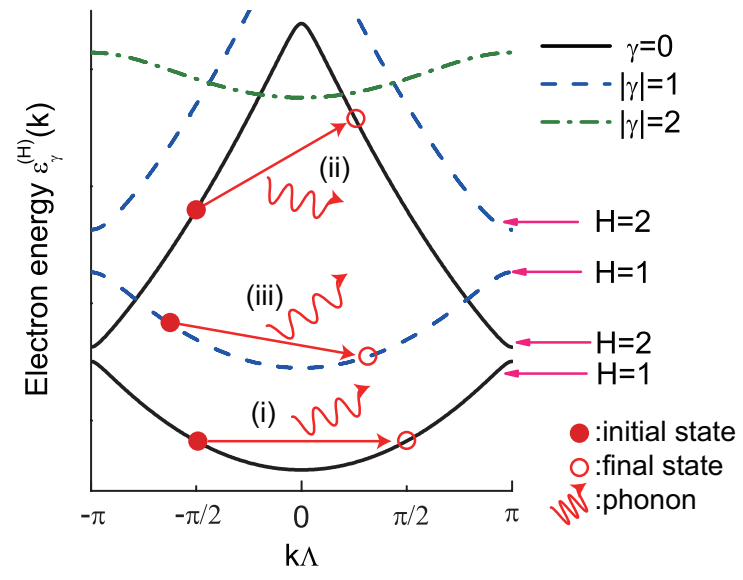

FIG. 5. (Color online) Electron band structure and phonon emission process for several bands. The intra-band electron scatterings of (i), (ii), and (iii) within the electronic band labeled by $(|\gamma|, H)=(0,1),(0,2)$, and $(1,1)$ are depicted, respectively.

Finally, we arrive at the interaction Hamiltonian in the reciprocal-space representation by substituting Eqs. (13) and (17) into Eq. (11):

$$
\mathcal{H}_{e-p h}=\sum_{\sigma} \sum_{P_{i}, P_{f}} \sum_{J} g_{J}^{P_{i}, P_{f}} a_{P_{f}, \sigma}^{\dagger} a_{P_{i}, \sigma}\left(b_{J}+b_{-J}^{\dagger}\right),
$$

where the labels $J=(m, q, M)$ and $P_{j}=\left(\gamma_{j}, k_{j}, H_{j}\right)$ indicate the phonon and electron modes, respectively. The quantity $g_{J}^{P_{i}, P_{f}}$ describes the strength of the electronphonon interaction through which electrons are scattered from initial states $(j=i)$ to final ones $(j=f)$; several scattering processes are illustrated in Fig. [5. We can prove that

$$
\begin{gathered}
g_{J}^{P_{i}, P_{f}}=D c_{J} \sum_{\alpha}\left[A_{\alpha r}^{(J)}\left(\frac{l+1}{R_{0}}\right) K_{l-1, G, 0}^{P_{i}, P_{f}, m, q}\right. \\
\left.+A_{\alpha z}^{(J)}\left(\frac{i m}{R_{0}}\right) K_{l-1, G, 0}^{P_{i}, P_{f}, m, q}+A_{\alpha z}^{(J)} i(q-G) K_{l, G, 1}^{P_{i}, P_{f}, m, q}\right],
\end{gathered}
$$

where

$$
\begin{aligned}
K_{l, G, p}^{P_{i}, P_{f}, m, q} & =\sum_{\tilde{l}} \delta_{\gamma_{i}+m, \gamma_{f}} \delta_{k_{i}+q-k_{f}, 2 \pi \tilde{l} / \Lambda} \\
& \times \frac{1}{\Lambda} \sum_{G^{\prime}, G^{\prime \prime}} C_{\gamma_{f}, k_{f}-G^{\prime}}^{\left(H_{f}\right) *} C_{\gamma_{i}, k_{i}-G^{\prime \prime}}^{\left(H_{i}\right)} \\
& \times \int_{0}^{s} d z w^{1+p}\left(\frac{R}{R_{0}}\right)^{l_{p}} e^{i\left(2 \pi \tilde{l} / \Lambda-G+G^{\prime}-G^{\prime \prime}\right) \xi(z)},
\end{aligned}
$$

and $C_{\gamma, k-G}^{(H)}$ is a Fourier expansion coefficient of $\psi_{\gamma, k}^{(H)}$ as given by Eq. (B3). In Eq. (20), the integer $\tilde{l}$ is introduced to let $k_{i}$ and $k_{f}$ be in the first Brillouin zone, i.e., $k_{j} \Lambda \in$ $[-\pi, \pi]$. 

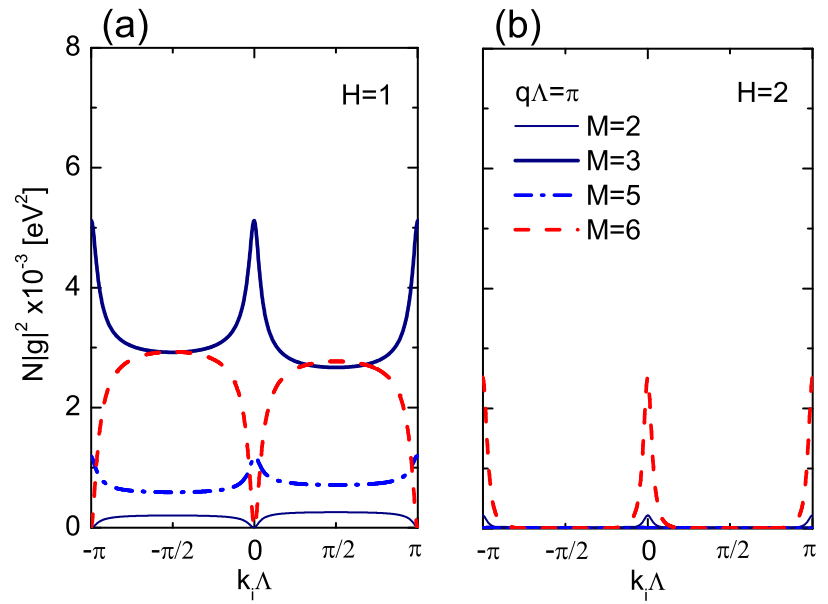

FIG. 6. (Color online) Coupling strength $|g|^{2}$ in the case of $\gamma=0$. The dependences on the electron's initial wavenumber $k_{i}$ are displayed, while the phonon mode parameters $m=0$ and $q \Lambda=\pi$ are fixed.

\section{COUPLING STRENGTH}

The coupling strength $\left|g_{J}^{P_{i}, P_{f}}\right|^{2}$ for various conditions of $J(=m, q, M)$ and $P_{j}\left(=\gamma_{j}, k_{j}, H_{j}\right)$ with $j=i$ or $f$ has been systematically calculated, only a part of which are presented in Figs. 6 and 7. In all the figures, we set $m \equiv 0, q \Lambda \equiv \pi, \gamma_{i}=\gamma_{f}(\equiv \gamma)$, and $H_{i}=H_{f}(\equiv H)$, since the intra-band electron scatterings that satisfy these conditions (see Fig. 5) were found to yield significantly large values of $\left|g_{J}^{P_{i}, P_{f}}\right|^{2}$. Hereafter, the suffixes in $g_{J}^{P_{i}, P_{f}}$ are omitted for simple notation.

First, we look into $|g|^{2}$ for $\gamma=0$ and $H=1$, in which only the process (i) depicted in Fig. 5 is relevant. Figure 6(a) shows $|g|^{2}$ as a function of the initial wavenumber $k_{i}$ for four different $M$ s, i.e., $P_{i}=\left(0, k_{i}, 1\right)$, $P_{f}=\left(0, k_{i}+\pi / \Lambda, 1\right)$, and $J=(0, \pi / \Lambda, M)$. The contributions from $M=1,4$ to $|g|^{2}$ vanish at every $k_{i}$, because these twisting modes give $\nabla \cdot \tilde{\boldsymbol{u}}=0$. For almost all $k_{i},|g|^{2}$ with $M=3$ takes the largest value, while $|g|^{2}$ with $M=6$ competes within limited ranges around $k_{i} \Lambda \simeq \pm \pi / 2$. The primary contributions to $|g|^{2}$ from the phonon modes of $M=3,6$ can be explained by their longitudinal nature. As shown in Fig. 4, the displacement vectors in the two modes $(\mathrm{C}$ and $\mathrm{F})$ are mostly tangential to the modulated cylindrical surface, thus yielding large values of $\nabla \cdot \tilde{\boldsymbol{u}}$. This results in the enhancement of the electron-phonon coupling as follows from the expression of $\mathcal{H}_{\mathrm{e}-\mathrm{ph}}$ given in Eq. (18).

The profile of $|g|^{2}$ drastically changes when electrons stay at upper bands of $H \geq 2$. Figure 6(b) shows the contribution to $|g|^{2}$ from the process (ii) in Fig. [5 i.e., $H=2$ and $\gamma=0$. In this case, $|g|^{2}$ is strongly depressed except for a few peaks at $k_{i} \Lambda=0, \pm \pi$. If we increase $H$ further, the peaks diminish rapidly and $|g|^{2}$ disappears

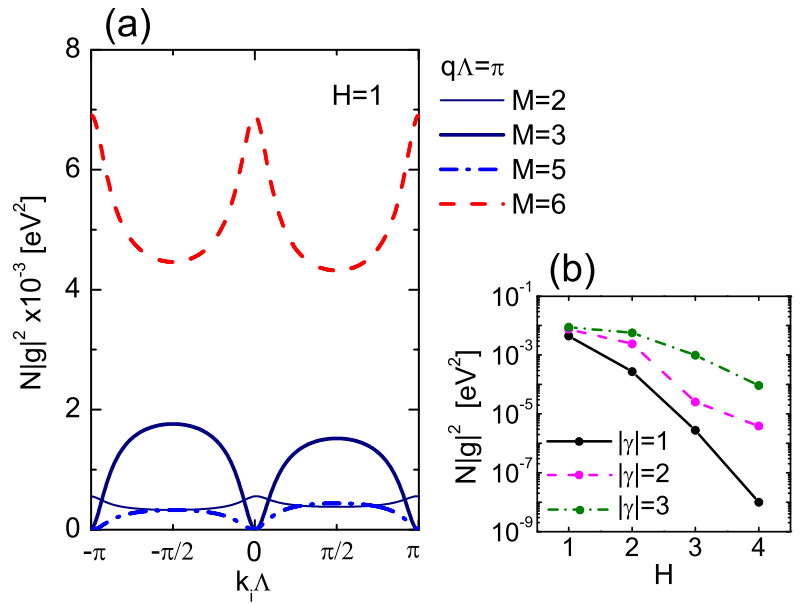

FIG. 7. (Color online) (a) The $k_{i}$-dependence of $|g|^{2}$ for $|\gamma|=$ 1. (b) Rapid decrease in $|g|^{2}$ of $M=6$ at $k_{i} \Lambda=\pi / 2$ with the electron band index $H$.

for all $k_{i}$. This disappearance is in contrast with $|g|^{2}$ for $H=1$, where every $k_{i}$ give finite values of $|g|^{2}$. We thus conclude that, in the modulated cylinders, significant values of $|g|^{2}$ can be obtained only when the coupled electrons belong to the lowest band of $H=1$.

Next, we pay attention to the case of $\gamma \neq 0$. Figure 7(a) shows $|g|^{2}$ s for $|\gamma|=1$ and $H=1$ [the process (iii) in Fig. 囵, in which $|g|^{2}$ with $M=6$ is the largest for all $k_{i}$ and that with $M=3$ shows a modest value. We have confirmed that as $H$ increases, every curves of $|g|^{2}$ go downward, which result in profiles of $|g|^{2}$ analogous to Fig. 6(b). Similar depression of $|g|^{2}$ with increasing $H$ have been observed for $|\gamma| \geq 2$, as shown in Fig. 7(b). We can say that for arbitrary $\gamma,|g|^{2}$ for $M=3$ or 6 has significant value only when both two coupled electrons reside at a small $H$ branch.

\section{PHYSICAL CONSEQUENCES}

\section{A. DOMINANT MODE COUPLING}

Our findings of $|g|^{2}$ in Sec $\nabla$ give an important suggestion for addressing the property of the possible Peierls transition in the $1 \mathrm{D}_{60}$ polymers. As is well known, the Peierls transition temperature $T_{c}$ in general 1D systems is dependent on the coupling strength $|g|^{2}$; the larger value $|g|^{2}$ arises, the higher $T_{c}$ we observe 30]. It follows from Sec. V that $|g|^{2}$ becomes significant only when the electrons lie at a small $H$ branch and a specific phonon mode of $M=3$ or 6 is involved. All the rest combination of electronic and phononic modes give slight contribution to the Peierls transition in the $1 \mathrm{D} \mathrm{C}_{60}$ polymers. In this context, we have succeeded to determine what specific modes (electron and phonon) in the $1 \mathrm{D}_{60}$ polymers should be 
(a)

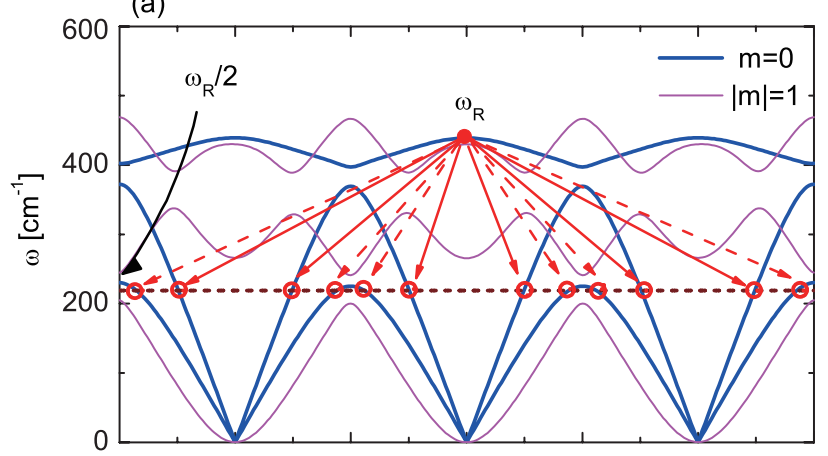

(b)

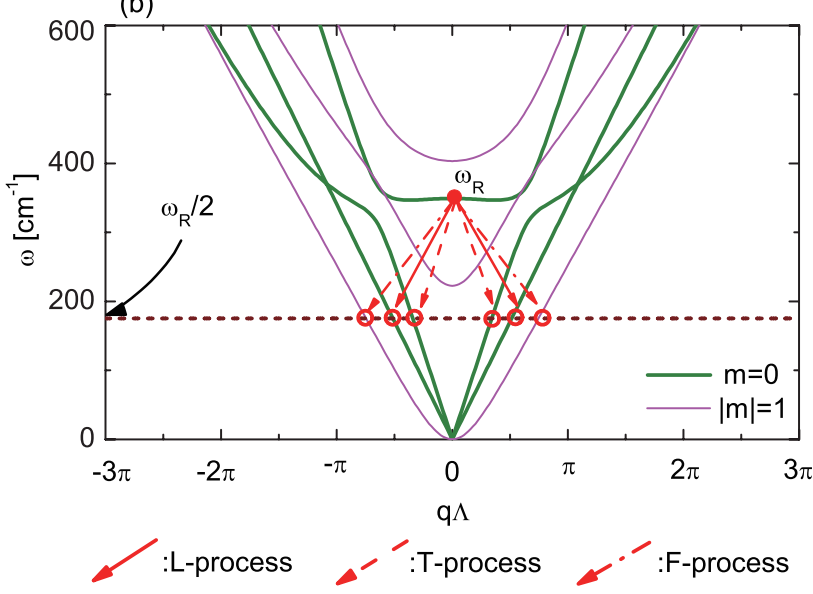

FIG. 8. (Color online) Allowed relaxation process of RBMs for (a) the $1 \mathrm{D} \mathrm{C}_{60}$ polymer and (b) a straight tube. The L-, T, and F-processes suggest the RBM's relaxation in which the post-decay modes are the longitudinal, twisting, and flexural modes, respectively.

responsible for the Peierls transition endowed with a feasible value of $T_{c}$. Quantitative evaluation of $T_{c}$ for the $1 \mathrm{D}$ $\mathrm{C}_{60}$ polymers is possible via the standard Green's function technique 31] incorporated with our formulation of $|g|^{2}$ given in Eqs. (19) and (20). The calculated results of $T_{c}$ as well as the comparison with experiments will be discussed elsewhere.

We emphasize that the present formulation of $|g|^{2}$ is broadly applicable to the study of the radius modulation effects on the physical properties of $1 \mathrm{D} \mathrm{C}_{60}$ polymers. The application should include the electrical resistivity analyses. In general, the low-temperature resistivity of nanomaterials is dependent on temperature and the Fermi energy, and the dependences are governed largely by the magnitude of $|g|^{2}$. Hence, our formulation of $|g|^{2}$ will be helpful to estimate the low-temperature resistivity of the $1 \mathrm{D} \mathrm{C}_{60}$ polymers that remains untouched to date. In addition, artificial control of the Fermi level by chemical doping or bias voltage application may enable to obtain a desired magnitude of $|g|^{2}$, leading to an efficient means of developing next-generation nanodevices.

Besides the modulated cylinder problem, an extension of the present theory to three dimensional counterparts (e.g., the Mackay crystal 32] and the zeolite-templated carbon [33]) is quite interesting. The Mackay crystal is a three-dimensional carbon network; three infinite tubes intersect smoothly in the unit cell, resulting in a triply periodic minimal surface. Due to the intriguing geometry and functionality [34], it has attracted interest since the first hypothesis. We speculate that in the Mackay crystal, only specific phonon modes cause large deformation potential similarly to the present system, since each constituent tube in the three dimensional minimal surface can be regarded as a radius-modulated tube along the axial direction. Electronic structures of the periodic minimal surface have been already clarified [35-37], and thus employing the results to obtain the electron-phonon interaction Hamiltonian will yield a conclusion of this issue.

\section{B. RADIAL BREATHING MODE RELAXATION}

Another possible consequence of the periodic radius modulation in $1 \mathrm{D}_{60}$ polymers is a considerable decrease in the relaxation time of radial breathing modes (RBMs) compared with that in carbon nanotubes [38]. We have numerically found that the phononic mode labeled by $(m, q, M)=(0,0,3)$ is a RBM, which is active on the resonant Raman scattering. The lifetime of a RBM is mainly determined by phonon-phonon scatterings which lead to a phonon decay [38]. When the number of the allowed decay process is large, the corresponding phonon mode has a short life. This indeed holds true for the RBM of the $1 \mathrm{D} \mathrm{C}_{60}$ polymers, as demonstrated below.

Figures 8(a) and 8(b) illustrate the RBM decay processes in the $1 \mathrm{D} \mathrm{C}_{60}$ polymer and the straight tube, respectively. Allowed decay processes are represented by slanted arrows that extend from the RBM (indicated by $\omega_{R}$ ) to low-lying acoustic modes having the eigenfrequency $=\omega_{R} / 2$ : The post-decay states locate at the intersections of the horizontal dotted line $\left(=\omega_{R} / 2\right)$ and dispersion curves. Note that the decay process is constrained by the conservation laws of energy, wavenumber, and angular momentum. Therefore, a RBM having the eigenfrequency $\omega_{R}$ decays into a pair of acoustic phonons with $\omega_{m, q, M}$ and $\omega_{-m,-q+G, M}$ that satisfy $\omega_{R}=\omega_{m, q, M}+\omega_{-m,-q+G, M}$; here, $G=0$ for normal processes and $G \neq 0$ for umklapp processes. It is found that in the straight tube $\left(\omega_{R}=350 \mathrm{~cm}^{-1}\right)$, there are four decay processes: L-, T-, and doubly-degenerate Fprocesses (i.e., $\omega_{1, q, 1}+\omega_{-1,-q, 1}$ and $\omega_{1,-q, 1}+\omega_{-1, q, 1}$ ), where $\mathrm{L}, \mathrm{T}$, and $\mathrm{F}$ mean that the post-decay modes are the longitudinal, twisting, and flexural modes, respectively. All the processes belong to the normal process (i.e., $G=0$ ). On the other hand, the RBM of the polymer $\left(\omega_{R}=440 \mathrm{~cm}^{-1}\right)$ can decay through both the normal and umklapp processes $(G \neq 0)$ involving L- and T-processes. Due to many choices of $G$ available, the number of decay process in the polymer is much larger than that in the straight tube, although the F-process in the polymer is forbidden as the dispersion curves with 
$|m|=1$ has the frequency gap around $\omega_{R} / 2$. In other words, the zone-folding in the phonon dispersion results in a considerable increase in the number of the decay process in the polymer case, which leads to a broadening of the RBM peak linewidth. It is known that at room temperature, the linewidth of the RBM peak in carbon nanotubes is $\sim 1 \mathrm{~cm}^{-1}$ independently of the tube radius [38]. Hence, that in the $1 \mathrm{D} \mathrm{C}_{60}$ polymer is expected to be larger than $1 \mathrm{~cm}^{-1}$ as a result of the periodic radius modulation. The theoretical estimation of the linewidth will be carried out in our future work.

\section{CONCLUSION}

We have investigated the low-frequency phonon states and their coupling with electrons in the $1 \mathrm{D}$ peanutshaped $\mathrm{C}_{60}$ polymers along the continuum approximation. It has been found that the phonon dispersion relations exhibit the zone-folding effect due to the periodic radial modulation. The electron-phonon interaction was formulated in line with the deformation potential theory, and the obtained formula for the coupling strength allows us to identify which phonon modes should be responsible for the Peierls transition observed in the prior experiment. The present formulation for the coupling strength has a wide applicability for estimating physical quantities of the systems, thus playing a crucial role in predicting untouched behaviors governed by the electron-phonon interaction.

\section{ACKNOWLEDGMENTS}

We would like to thank K. Yakubo, N. Nishiguchi, S. Mizuno and Y. Tanaka for helpful suggestions toward formulation of the elasticity theory. We also appreciate informative comments given by H. Suzuura and H. Yoshioka. This study was supported by a Grant-in-Aid for Scientific Research from the MEXT, Japan. HS acknowledgments the supports from Hokkaido Gas Co., Ltd. and The Sumitomo Foundation. A part of numerical simulations were carried out using the facilities of the Supercomputer Center, ISSP, University of Tokyo.

\section{Appendix A: EXPRESSIONS OF $\Gamma_{(\beta i)(\alpha l)}$}

The matrix elements $\Gamma_{(\beta i),(\alpha l)}$ introduced in Eq. (4) are represented as follows.

For $\Gamma_{(\beta i),(\alpha l)}$ with $i \equiv r$, we have

$$
\begin{aligned}
\Gamma_{(\beta r),(\alpha r)} & =\frac{\lambda}{\rho V} \int \Phi_{\beta,+}^{*} \Phi_{\alpha,+} d V+\frac{\mu}{\rho V} \int\left(2 \partial_{r} \phi_{\beta}^{*} \partial_{r} \phi_{\alpha}+\frac{1}{r^{2}} \partial_{\theta} \phi_{\beta}^{*} \partial_{\theta} \phi_{\alpha}+\partial_{z} \phi_{\beta}^{*} \partial_{z} \phi_{\alpha}+\frac{2}{r^{2}} \phi_{\beta}^{*} \phi_{\alpha}\right) d V, \\
\Gamma_{(\beta r),(\alpha \theta)} & =\frac{\lambda}{\rho V} \int \Phi_{\beta,+}^{*} \frac{1}{r} \partial_{\theta} \phi_{\alpha} d V+\frac{\mu}{\rho V} \int\left(\frac{1}{r} \partial_{\theta} \phi_{\beta}^{*} \Phi_{\alpha,-}+\frac{2}{r^{2}} \phi_{\beta}^{*} \partial_{\theta} \phi_{\alpha}\right) d V, \\
\Gamma_{(\beta r),(\alpha z)} & =\frac{\lambda}{\rho V} \int \Phi_{\beta,+}^{*} \partial_{z} \phi_{\alpha} d V+\frac{\mu}{\rho V} \int \partial_{z} \phi_{\beta}^{*} \partial_{r} \phi_{\alpha} d V .
\end{aligned}
$$

For $\Gamma_{(\beta i),(\alpha l)}$ with $i \equiv \theta$,

$$
\begin{aligned}
\Gamma_{(\beta \theta),(\alpha r)} & =\frac{\lambda}{\rho V} \int \frac{1}{r} \partial_{\theta} \phi_{\beta}^{*} \Phi_{\alpha,+} d V+\frac{\mu}{\rho V} \int\left(\frac{2}{r^{2}} \partial_{\theta} \phi_{\beta}^{*} \phi_{\alpha}+\Phi_{\beta,-}^{*} \frac{1}{r} \partial_{\theta} \phi_{\alpha}\right) d V, \\
\Gamma_{(\beta \theta),(\alpha \theta)} & =\frac{\lambda}{\rho V} \int \frac{1}{r^{2}} \partial_{\theta} \phi_{\beta}^{*} \partial_{\theta} \phi_{\alpha} d V+\frac{\mu}{\rho V} \int\left(\frac{2}{r^{2}} \partial_{\theta} \phi_{\beta}^{*} \partial_{\theta} \phi_{\alpha}+\Phi_{\beta,-}^{*} \Phi_{\alpha,-}+\partial_{z} \phi_{\beta}^{*} \partial_{z} \phi_{\alpha}\right) d V, \\
\Gamma_{(\beta \theta),(\alpha z)} & =\frac{\lambda}{\rho V} \int \frac{1}{r} \partial_{\theta} \phi_{\beta}^{*} \partial_{z} \phi_{\alpha} d V+\frac{\mu}{\rho V} \int \frac{1}{r} \partial_{z} \phi_{\beta}^{*} \partial_{\theta} \phi_{\alpha} d V .
\end{aligned}
$$

For $\Gamma_{(\beta i),(\alpha l)}$ with $i \equiv z$,

$$
\begin{aligned}
\Gamma_{(\beta z),(\alpha r)} & =\frac{\lambda}{\rho V} \int \partial_{z} \phi_{\beta}^{*} \Phi_{\alpha,+} d V+\frac{\mu}{\rho V} \int\left(\partial_{r} \phi_{\beta}^{*} \partial_{z} \phi_{\alpha}\right) d V \\
\Gamma_{(\beta z),(\alpha \theta)} & =\frac{\lambda}{\rho V} \int \frac{1}{r} \partial_{z} \phi_{\beta}^{*} \partial_{\theta} \phi_{\alpha} d V+\frac{\mu}{\rho V} \int \frac{1}{r} \partial_{\theta} \phi_{\beta}^{*} \partial_{z} \phi_{\alpha} d V \\
\Gamma_{(\beta z),(\alpha z)} & =\frac{\lambda}{\rho V} \int \partial_{z} \phi_{\beta}^{*} \partial_{z} \phi_{\alpha} d V+\frac{\mu}{\rho V} \int\left(\partial_{r} \phi_{\beta}^{*} \partial_{r} \phi_{\alpha}+\frac{1}{r^{2}} \partial_{\theta} \phi_{\beta}^{*} \partial_{\theta} \phi_{\alpha}+2 \partial_{z} \phi_{\beta}^{*} \partial_{z} \phi_{\alpha}\right) d V .
\end{aligned}
$$

Here, we have used the following expression

$$
\Phi_{p, \pm}=\partial_{r} \phi_{p} \pm \frac{\phi_{p}}{r} .[p=\alpha, \beta]
$$

The volume integral and the basis function $\phi_{\alpha(\beta)}$ are defined in Eqs. (8) and (9) in Sec. IIB, respectively. 


\section{Appendix B: ELECTRON EIGENSTATES}

In this Appendix, we derive electronic states in the cylindrical tube with varying radius. We have assumed that the tube thickness is small enough to suppress electron's excitation in the normal direction. The same assumption works well for treating the interaction between electrons in both $1 \mathrm{D} \mathrm{C}_{60}$ polymers [10] and twodimensional curved thin films [1]. Due to the rotational symmetry of the tube, the wavefunction becomes $\psi(\theta, z)=\left(e^{i \gamma \theta} / \sqrt{2 \pi}\right) \chi_{\gamma}(z)$ with the electronic angular quantum number $\gamma$. The Schrödinger equation for $\chi_{\gamma}(z)$ is written as [10, 29]

$$
-\frac{\hbar^{2}}{2 m^{*}}\left[\frac{1}{R w} \partial_{z}\left(\frac{R}{w} \partial_{z}\right)+U_{\gamma}(z)\right] \chi_{\gamma}(z)=\epsilon_{\gamma} \chi_{\gamma}(z) .
$$

Here, $m^{*}$ is the effective mass and $U_{\gamma}(z)$ is an effective potential expressed by

$$
U_{\gamma}(z)=-\frac{\gamma^{2}}{R^{2}}+\left(\frac{w^{2}-R \partial_{z}^{2} R}{2 R w^{3}}\right)^{2}+\frac{\partial_{z}^{2} R}{R w^{4}} .
$$

Since $R(z)$ and $w(z)$ are periodic as shown by Eqs. (6) and (17), $U_{\gamma}(z)$ is also periodic, i.e., $U_{\gamma}(z)=U_{\gamma}(z+s)$. Thus, it follows from Bloch's theorem that

$$
\psi_{\gamma, k}^{(H)}(\theta, z)=\frac{e^{i \gamma \theta}}{\sqrt{2 \pi}}\left[\frac{1}{\sqrt{N \Lambda R}} \sum_{G_{e}} C_{\gamma, k-G_{e}}^{(H)} e^{i\left(k-G_{e}\right) \xi(z)}\right],
$$

where $G_{e}=2 \pi n_{e} / \Lambda\left(n_{e}=0, \pm 1, \pm 2, \cdots\right)$.
[1] A. M. Rao, P. Zhou, K.-A. Wang, G. T. Hager, J. M. Holden, Y. Wang, W.-T. Lee, X.-X, Bi, P. C. Eklund, D. S. Cornett, D. S. Duncan, and I. J. Amster, Science 259, 955 (1993).

[2] A. M. Rao and P. C. Eklund, Fullerene Polymers and Fullerene Polymer Composites (Springer Berlin, 2000).

[3] J. Onoe, T. Nakayama, M. Aono, and T. Hara, Appl. Phys. Lett. 82, 595 (2003).

[4] J. Onoe, T. Ito, and S. Kimura, J. Appl. Phys. 104, 103706 (2008).

[5] G. Wang, Y. Li, and Y. Huang, J. Phys. Chem. B 109, 10957 (2005).

[6] T. A. Beu, J. Onoe, and A. Hida, Phys. Rev. B 72, 155416 (2005).

[7] T. A. Beu and J. Onoe, Phys. Rev. B 74, 195426 (2006).

[8] H. Nakayama, T. Ono, H. Goto, and K. Hirose, Sci. Technol. Adv. Mater. 8, 196 (2007).

[9] A. Takashima, J. Onoe, and T. Nishii, J. Appl. Phys. 108, 033514 (2010).

[10] H. Shima, H. Yoshioka, and J. Onoe, Phys. Rev. B 79, 201401(R) (2009); Physica E 42, 1151 (2010).

[11] S. Ono and H. Shima, Phys. Rev. B 79, 235407 (2009); Physica E 42, 1224 (2010).

[12] Y. Toda, S. Ryuzaki, and J. Onoe, Appl. Phys. Lett. 92, 094102 (2008).

[13] C. Kittel, Introduction to Solid State Physics (Wiley, Hoboken, NJ, 2005), 8th ed.

[14] N. Nishiguchi, Phys. Rev. B 52, 5279 (1995).

[15] S. Mizuno and N. Nishiguchi, J. Phys.:Condens. Matter 21, 195303 (2009).

[16] D. Kahn, K. W. Kim, and M. A. Stroscio, J. Appl. Phys. 89, 5107 (2001).

[17] G. D. Mahan, Phys. Rev. B 65, 235402 (2002).

[18] H. Suzuura and T. Ando, Phys. Rev. B 65, 235412 (2002).

[19] L. Chico, R. Perez-Alvarez, and C. Cabrillo, Phys. Rev. B 73, 075425 (2006).

[20] G. Li, G. A. Lamberton, Jr., and J. R. Gladden, J. Appl. Phys. 104, 033524 (2008).
[21] L. D. Landau and E. M. Lifshitz, Elasticity Theory (Pergamon, New York, 1986).

[22] W. M. Visscher, A. Migliori, T. Bell, and R. Reinert, J. Acoust. Soc. Am. 90, 2154 (1991).

[23] H. Shima and T. Nakayama, Higher Mathematics for Physics and Engineering (Springer-Verlag, 2010).

[24] D. Gunlycke, H. M. Lawler, and C. T. White, Phys. Rev. B 77, 014303 (2008).

[25] J. Zimmermann, P. Pavone, and G. Cuniberti, Phys. Rev. B 78, 045410 (2008).

[26] E. H. Hwang and S. Das Sarma, Phys. Rev. B 77, 115449 (2008).

[27] R. Leturcq, C. Stamper, K. Inderbitzin, L. Durrer, C. Heirold, E. Mariani, M. G. Schultz, F. von Oppen, and K. Ensslin, Nat. Phys. 5, 327 (2009).

[28] N. Nishiguchi, Y. Ando, and M. N. Wybourne, J. Phys.: Condens. Matter 9, 5751 (1997).

[29] N. Fujita, J. Phys. Soc. Jpn. 73, 3115 (2004).

[30] G. Grüner, Density Waves in Solids (Addison-Wesley, Reading, MA, 1994).

[31] A. A. Abrikosov, L. G. Gorkov, and I. E. Dzyaloshinski, Methods of Quantum Field Theory in Statistical Physics (Dover, New York,1963)

[32] A. L. Mackay and H. Terrones, Nature (London) 352, 762 (1991).

[33] K. Takai, T. Suzuki, T. Enoki, H. Nishihara, and T. Kyotani, Phys. Rev. B 81, 205420 (2010).

[34] N. Park, M. Yoon, S. Berber, J. Ihm, E. Osawa, and D. Tománek, Phys. Rev. Lett. 91, 237204 (2003).

[35] H. Aoki, M. Koshino, D. Takeda, H. Morise and K. Kuroki Phys. Rev. B 65, 035102 (2001).

[36] M. Koshino and H. Aoki, Phys. Rev. B 71, 073405 (2005).

[37] N. Fujita and O. Terasaki, Phys. Rev. B 72, 085459 (2005).

[38] R. Rao, J. Menendez, C. D. Poweleit, and A. M. Rao, Phys. Rev. Lett. 99, 047403 (2007). 